\title{
Perfil de saúde bucal em escolares residentes em uma região endêmica de fluorose dental
}

\section{Oral health profile of schoolchildren living in regions with endemic dental fluorosis Perfil de salud bucal en escolares residentes en una región endémica de fluorosis dental}

\section{Recebido: 18/04/2017 \\ Aprovado: 21/12/2017 \\ Publicado: 05/04/2018}

\section{Consuelo Fernanda Macedo de Souza ${ }^{1}$ Teodomiro Dutra de Abreu² Gabrielle Stella Dantas Barbosa ${ }^{3}$ Cynára Liane Jales Ataíde de Melo ${ }^{4}$ Maria Soraya Pereira Franco Adriano 5}

Este estudo teve como objetivo avaliar a saúde oral de escolares que vivem em área endêmica de fluorose dental. A região apresenta em sua água de consumo humano uma concentração de 5,16 mg/L de flúor. Esta é uma pesquisa do tipo observacional, transversal, de campo, realizada no município de São João do Rio do Peixe (PB) no ano de 2016. Dois examinadores treinados realizaram o exame clínico em escolares de 6 a 8 anos. Foram utilizados os índices de CPO-d, índice periodontal (PSR) e índice de Dean para avaliação de cárie dentária, saúde periodontal e grau de fluorose, respectivamente. Foram analisados 13 alunos com idades entre 6 e 8 anos. Os índices CPO-d e Ceo-d apresentaram valores médios de 1,0 (DP=1) e 2,6 (DP=3,5) respectivamente. Com relação aos dentes permanentes viu-se que 38,5\% dos voluntários apresentaram índice CPO-d 0, enquanto 7,7\% apresentavam pelos menos três elementos dentários afetados de alguma forma pela doença cárie. Com relação ao Ceo-d, 30,8\% apresentaram pelo menos 4 elementos acometidos pela doença. 0 diagnóstico de fluorose pelo índice TF mostrou que todos os voluntários apresentaram certo grau de fluorose. Conclui-se que as crianças residentes na região da Vila do Brejo apresentam cárie ativa, fluorose dentária em graus acima de 2 (índice TF) e presença de sangramento gengival.

Descritores: Fluorose dentária; Índice CPO; Gengivite.

This study aimed at evaluating the oral health of schoolchildren who live in an area which is endemic for dental fluorosis. The region presents a concentration of $5.16 \mathrm{mg} / \mathrm{L}$ of fluorine in its water for human consumption. This is an observational, cross-sectional, field research, conducted in the city of São José do Rio do Peixe (PB), in 2016. Two trained examiners analyzed the clinical exam of schoolchildren from 6 to 8 years of age. The CPO-d index, the periodontal index (PSR) and the Dean index to evaluate dental cavities, periodontal health and fluorosis levels, respectively. 13 students from 6 to 8 years of age were analyzed. The CPO-d and Ceo-d presented average levels of $1.0(\mathrm{DP}=10)$ and $2.6(\mathrm{DP}=3.5)$, respectively. Regarding their permanent teeth, it was found that $38.5 \%$ of volunteers presented a CPO-d level of 0 , while $7.7 \%$ presented at least three dental elements affected in some level by tooth decay. Regarding the Ceo-d, 30.8\% presented at least 4 elements affected by this disease. The fluorosis diagnostic from the TF index showed that all volunteers presented some level of fluorosis. It was concluded that the children from the Vila do Brejo region present active cavities, dental fluorosis in degrees above 2 (TF index) and the presence of gingival bleeding.

Descritores: Fluorose dentária; Índice CPO; Gengivite.

Este estudio tuvo como objetivo evaluar la salud oral de escolares que viven en área endémica de fluorosis dental. La región presenta en su agua de consumo humano una concentración de 5,16 mg/L de flúor. Esta es una investigación del tipo observacional, transversal, de campo, realizada en el municipio de São João do Rio do Peixe (PB) en el año 2016. Dos examinadores entrenados realizaron el examen clínico en escolares de 6 a 8 años. Fueron utilizados los índices de CPO-d, índice periodontal (PSR) e índice de Dean para la evaluación de la caries dental, salud periodontal y grado de fluorosis, respectivamente. Fueron analizados 13 alumnos con edades entre 6 a 8 años. Los índices de CPO-d y Ceo-d presentaron valores promedio de 1,0 (DP=1) y 2,6 (DP=3,5) respectivamente. Con relación a los dientes permanentes se vio que 38,5\% de los voluntarios presentaron índice de CPO-d 0, mientras que $7,7 \%$ presentaban por lo menos tres elementos dentales afectados de alguna forma por la enfermedad caries. Con relación al Ceo-d, 30,8\% presentaron por lo menos 4 elementos afectados por la enfermedad. El diagnóstico de fluorosis por el índice TF mostró que todos los voluntarios presentaron cierto grado de fluorosis. Se concluye que los niños residentes en la región de Vila do Brejo presentan caries activa, fluorosis dental en grados por encima de 2 (índice TF) y presencia de sangramiento gingival.

Descriptores: Fluorosis dental; Índice CPO; Gingivitis.

1. Cirurgiã-Dentista. Especialista em Endodontia. Mestre em Odontologia. Doutoranda em Desenvolvimento e Inovação Técnológica de Medicamentos pela Universidade Federal do Rio Grande do Norte (UFRN). Professora Adjunta do Centro Universitário de João Pessoa (UNIPÊ), João Pessoa-PB, Brasil. ORCID: 0000-0003-3671-1106 E-mail: consuelofernanda79@hotmail.com

2. Cirurgião-Dentista. João Pessoa-PB, Brasil. ORCID:0000-0003-0190-6421 E-mail: teod.miro@gmail.com

3. Cirurgiã-Dentista. João Pessoa-PB, Brasil. ORCID:0000-0002-4498-553X E-mail: gaby-barbosa1@hotmail.com

4. Cirurgiã-Dentista. João Pessoa-PB, Brasil. ORCID:0000-0002-1564-8729 E-mail: cynara.88@hotmail.com

5. Cirurgiã-Dentista. Doutora em Biotecnologia em Saúde. Profa. Adjunta da Escola Técnica de Saúde da Universidade Federal da Paraíba. João Pessoa-PB, Brasil. ORCID:0000-0001-7888-4430 E-mail: msorayapf@hotmail.com 


\section{INTRODUÇÃO}

O uso do flúor é benéfico para a população quando em concentrações ideais, diminuindo a prevalência de cárie dentária. No entanto quando utilizado sistemicamente em concentrações elevadas e por longos períodos de tempo, pode levar à fluorose dentária e até mesmo à fluorose óssea, que é um quadro mais grave da doença ${ }^{1}$.

A fluorose dentária só ocorre quando há ingestão excessiva de fluoretos no período de formação dentária, enquanto que a fluorose óssea não apresenta um período especifico de susceptibilidade 2 .

A Organização Mundial da Saúde reconhece a importância da fluoretação da água no controle da cárie desde 1958. No entanto, essa fluoretação necessita ser controlada a depender da temperatura local, ou seja, o limite considerado ótimo para a concentração de fluoretos pode variar entre $0,7 \mathrm{mg} / \mathrm{L} \mathrm{e} 1,2 \mathrm{mg} / \mathrm{L}^{2}$.

No Brasil existe uma grande quantidade de pessoas, principalmente da zona rural, que usam como água de consumo aquela proveniente de poços artesianos, que apresentam altas concentrações residuais de fluoretos, incorrendo no risco de fluorose dentária ou até mesmo óssea ${ }^{2}$.

As pessoas que residem em região de altas concentrações de fluoretos em sua água de consumo desenvolvem a fluorose dentária e não se encontram livres da cárie dentária, uma vez que o fluoreto não vai exercer influência sobre os fatores etiológicos (biofilme, estrutura dentária, dieta ou bactérias). Daí a importância de estudar o perfil de saúde bucal de pacientes residentes em áreas de fluorose endêmica com o objetivo de alertar os profissionais para a prevalência de outros problemas orais de tais pacientes ${ }^{1}$.

Assim, este estudo teve como objetivo avaliar a saúde oral de escolares que vivem em área endêmica de fluorose dental.

\section{MÉTODO}

Esta pesquisa se realizou no município de São João do Rio do Peixe que está localizado no alto sertão paraibano, ficando a $480 \mathrm{~km}$ de João Pessoa, e apresentando população estimada em 17.929 habitantes segundo o IBGE (Instituto Brasileiro de Geografia e Estatística).

0 presente estudo foi realizado em 2016 na Vila do Brejo, zona rural deste município, situada a $10 \mathrm{~km}$ da sede do município. De acordo com o IBGE, São João do Rio do Peixe possui uma densidade demográfica de $38,36\left(\mathrm{hab} / \mathrm{km}^{2}\right.$ ), possuindo também uma área territorial de $474,4 \mathrm{~km}^{2}$, mostrando assim que é uma cidade com uma dimensão grande para um município do interior da Paraíba.

Foram avaliadas crianças entre 6 e 8 anos que moram na área desde o nascimento, caracterizando assim o uso crônico de fluoretos, e estudam na Escola Municipal de Educação Infantil e Ensino Fundamental Raimundo Rodrigues dos Santos. Foi escolhida essa localidade pelo fato de haver um alto índice de fluorose na população que mora na área.

Os exames clínicos foram realizados por dois avaliadores previamente calibrados sob iluminação natural na própria escola, após o consentimento do voluntário expresso através da assinatura dos termos de consentimento e assentimento, com uso de espelho bucal e sonda periodontal (OMS).

0 estudo foi do tipo observacional, transversal e de campo, e suas análises só tiveram início após aprovação no CEP (1.594.917).

\section{RESULTADOS}

Foram avaliados 13 alunos com idades entre 6 e 8 anos, sendo 46,2\% do sexo masculino e $53,8 \%$ do sexo feminino. Estavam matriculados na escola 19 alunos; no entanto, seis se recusaram a participar do estudo.

Os índices CPO-d e Ceo-d apresentaram valores médios de 1,0 (DP=1) e 2,6 $(\mathrm{DP}=3,5)$, respectivamente. Com relação aos dentes permanentes viu-se que $38,5 \%$ dos voluntários apresentaram índice CPO-d 0 , enquanto $7,7 \%$ apresentavam pelos menos três elementos dentários afetados de alguma forma pela doença cárie. No Ceo-d, 30,8\% tiveram ao menos 4 elementos acometidos pela doença (Gráficos 1 e 2). 


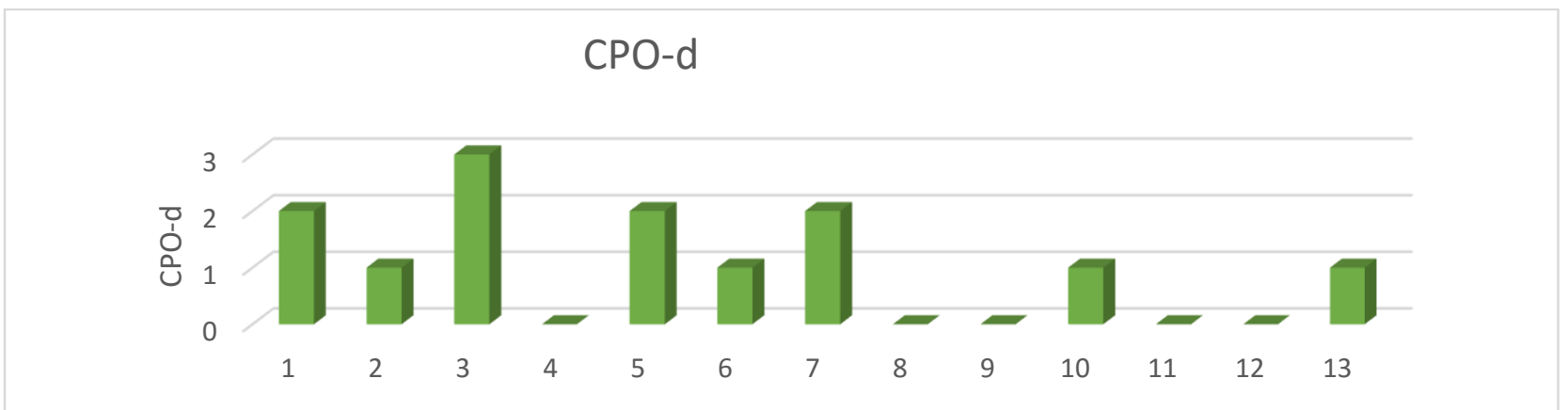

Gráfico 1: Índice CPO-d por voluntário. São José do Rio do Peixe, PB, 2016.

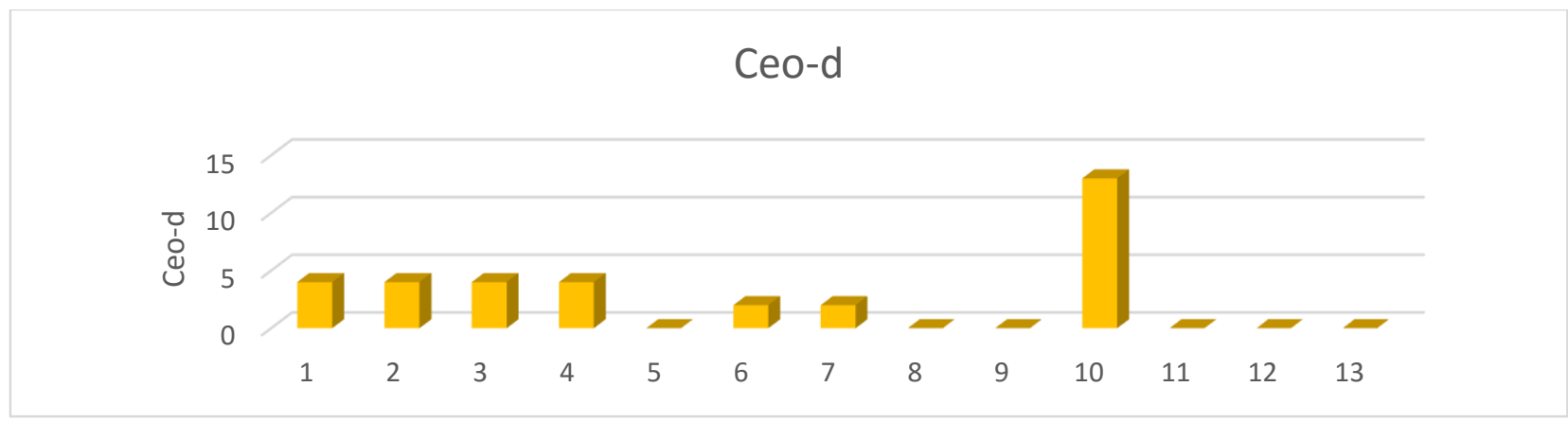

Gráfico 2: Índice Ceo-d por voluntário. São José do Rio do Peixe, PB, 2016.

Dentre as cáries ativas encontradas em realizado pelo índice TF, todos os voluntários elementos dentários, 13 foram identificadas apresentaram certo grau de fluorose, nos primeiros molares permanentes. 0 ilustrado pelo gráfico a seguir (Gráfico 3).0 diagnóstico quanto à presença de fluorose foi gráfico 4 correlaciona as três variáveis, CPO-d, Ceo-d e fluorose.

\section{Indice TF}

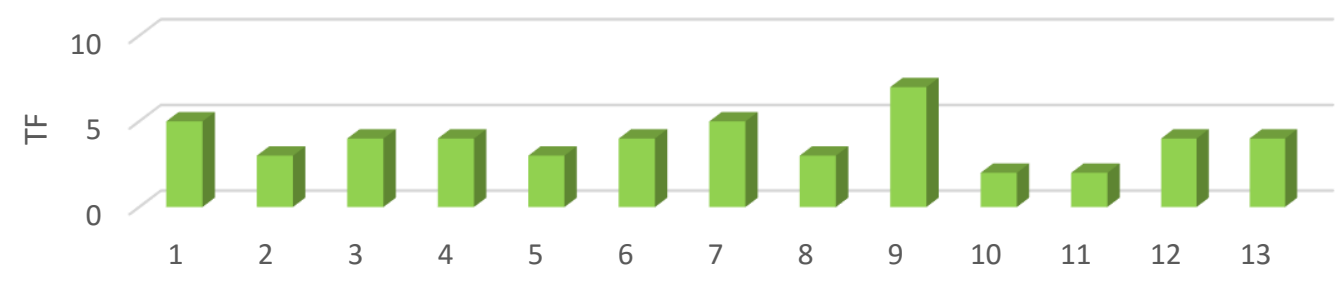

Gráfico 3: Índice TF por voluntário. São José do Rio do Peixe, PB, 2016.

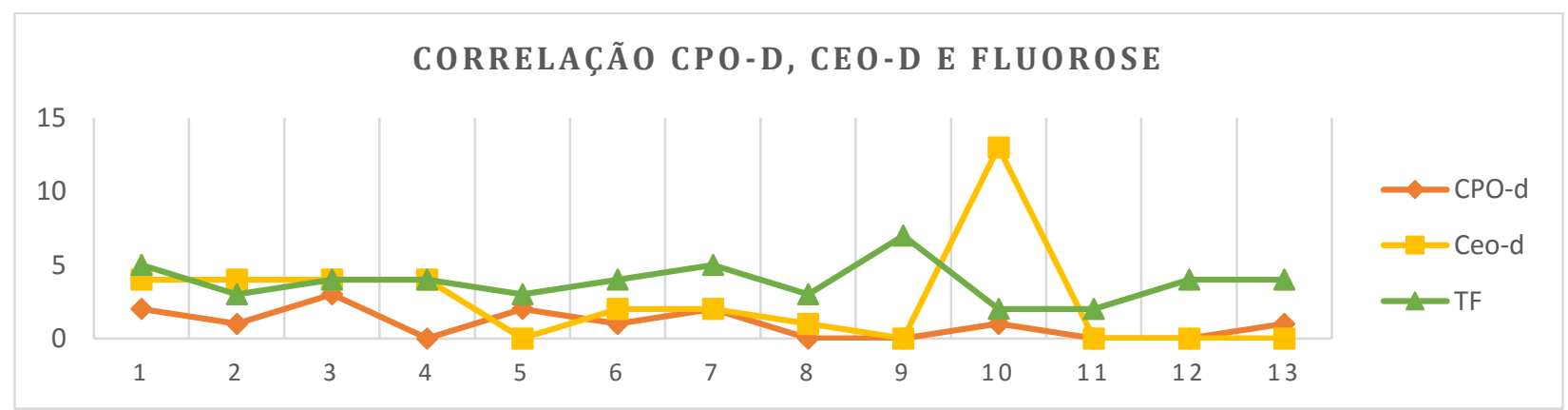

Gráfico 4. Correlação entre índice de cárie e fluorose. São José do Rio do Peixe, PB, 2016. 
Com relação à saúde periodontal, um voluntário apresentou dois sextantes da boca com periodontite moderada. Apenas três voluntários apresentaram condições periodontais saudáveis e nove voluntários apresentaram sangramento à sondagem (Tabela 1).

Tabela 1. Avaliação da saúde periodontal (PSR). São José do Rio do Peixe, PB, 2016.

\begin{tabular}{|ccccccc|}
\hline Voluntários & $* 17-16$ & 11 & $* 26-27$ & $* 36-37$ & $* 31$ & $* 4-46$ \\
\hline 1 & 1 & 0 & 1 & 0 & 0 & 0 \\
\hline 2 & 3 & 1 & 1 & 3 & 0 & 0 \\
\hline 3 & 0 & 0 & 0 & 0 & 1 & 0 \\
\hline 4 & 1 & 0 & 1 & 0 & 0 & 0 \\
\hline 5 & 0 & 0 & 0 & 0 & 0 & 0 \\
\hline 6 & 0 & 0 & 1 & 0 & 0 & 0 \\
\hline 7 & 1 & 1 & 1 & 0 & 1 & 0 \\
\hline 8 & 1 & 0 & 0 & 0 & 0 & 0 \\
\hline 9 & 1 & 0 & 1 & 1 & 1 & 0 \\
\hline 10 & 0 & 0 & 1 & 0 & 0 & 0 \\
\hline 11 & 0 & 0 & 0 & 0 & 0 & 0 \\
\hline 12 & 0 & 0 & 0 & 0 & 0 & 0 \\
\hline 13 & 0 & 0 & 0 & 0 & 0 & 0 \\
\hline
\end{tabular}

*Elementos dentais avaliados.

Foi constatado ainda que $100 \%$ dos voluntários afirmaram ter ciência de que havia manchas em seus dentes, 23,1\% afirmaram sentir desconforto com a presença dessas manchas, $69,2 \%$ diziam sentir vergonha de sorrir e 76,9\% gostariam de fazer tratamento para remover as manchas causadas pela fluorose.

\section{DISCUSSÃO}

A região nordeste do Brasil é caracterizada por possuir a maior parte do seu território influenciado pelo clima semiárido, cujas características são chuvas escassas devido à baixa precipitação anual. Por conta dessa escassez de água para consumo humano, a água de poço está se tornando uma importante estratégia para suprir as necessidades da população.

No entanto, a obtenção de águas subterrâneas sem caracterização e análise pode apresentar riscos à saúde humana, devido ao enriquecimento de minerais advindos da lixiviação do lençol freático e de outras fontes contaminantes.

Dentre os minerais que podem ser indesejáveis quando em moderada ou elevada concentração está o flúor. 0 flúor, apesar de ser uma das mais eficientes medidas preventivas contra a cárie dentária, está presente em concentrações elevadas nas águas de poços artesianos e, portanto, podem levar ao desenvolvimento de fluorose dentária ou óssea ${ }^{3}$.

Pelo menos três regiões no estado da Paraíba podem ser consideradas como região de risco para a fluorose dentária ${ }^{3}$. Todas estas áreas estão localizadas em locais de clima semiárido do estado e vários trabalhos mapearam e avaliaram o problema nessas regiões ${ }^{3,5}$.

A região de estudo alvo da presente pesquisa é uma dessas três regiões, e de acordo com uma pesquisa 6 , a região de Vila do Brejo apresenta uma concentração média de 5,16 ppm de flúor na água de consumo, sendo considerada endêmica em fluorose dentária e sujeita a casos de fluorose esquelética.

O flúor é o único elemento químico capaz de promover a prevenção de uma doença, a cárie dentária, e simultaneamente provocar o aparecimento de outra, a fluorose ${ }^{7}$.

Ao contrário da zona urbana do Nordeste, a prevalência de fluorose dentária em crianças do interior da Paraíba está praticamente relacionada apenas à 
concentração de flúor na água, uma vez que a água ainda é o principal veículo para a ingestão de flúor na região ${ }^{8,9}$.

0 percentual de crianças portadoras de fluorose dentária no interior da Paraíba é de $31 \%, 61 \%$ e $71,4 \%$ para concentrações de flúor na água de $0,10-0,70 ; 0,71-1,00$; e acima de 1,00 (até 3,50) mg/L, respectivamente 6 .

Em algumas localidades, a ingestão de flúor por crianças paraibanas tem se elevado a cada ano devido ao maior acesso aos cremes dentais fluoretados, e em algumas localidades, devido ao aumento eventual de alimentos industrializados 4,10 .

No presente estudo viu-se que $100 \%$ das crianças avaliadas apresentaram TF entre 2 e 7, estando 53\% entre os índices 4 e 5, que representam toda a superfície de esmalte branca e opaca com áreas de perda tecidual. Esse resultado corrobora outra investigação ${ }^{6}$ que examinou 650 crianças de áreas rurais da Paraíba, incluindo a Vila do Brejo, e observou fluorose dentária em $30,5 \%$ das crianças que consumiam água com concentrações abaixo de 0,7 ppm, $61,1 \%$ em crianças residentes nos locais onde a água de consumo possuía entre 0,7 e 1,0 ppm de flúor, e 71,4\% nas crianças que consumiam água com concentrações acima de 1,0 ppm de flúor.

A prevalência de fluorose está significativamente relacionada com a concentração de flúor na água de consumo. A severidade da doença variou entre TF 1 e 3 nos grupos de baixas concentrações de flúor e acima de 7 no restante 5 .

Outros lugares do mundo também têm altas de fluorose. Em estudo realizado na Índia em 2008 avaliou-se a prevalência e severidade de fluorose dentária em crianças entre 11 e 15 anos de idade. Foram avaliadas 1800 crianças, e se verificou que a prevalência de fluorose dentária era maior nas crianças que consumiam água de poço artesiano quando comparadas àquelas que consumiam água encanada ${ }^{11}$.

A fluorose dentária está relacionada à ingestão excessiva e prolongada de fluoreto durante a formação dos dentes. Assim, se pode inferir um período de susceptibilidade para a fluorose dentária nos dentes permanentes até os 5-6 anos de idade ${ }^{12}$.
As crianças avaliadas na presente pesquisa tinham entre 6 e 8 anos e eram residentes permanentes da região, assim como seus pais. Assim, todos com algum grau de fluorose nos dentes decíduos ou permanentes, e como utilizavam da água com alta concentração de flúor para consumo humano desde o nascimento, e inclusive na janela de susceptibilidade, serão acometidos pela fluorose também nos elementos permanentes. Aqueles indivíduos que passarem sua vida nessa região também estarão sob o risco de desenvolver a fluorose óssea.

0 esmalte com fluorose não é necessariamente mais rico em fluoreto, sendo na verdade hipomineralizado na sua subsuperfície, podendo ser observada elevada porosidade na região. Portanto, o dente fluorótico não é mais resistente ao desenvolvimento da cárie dentária - pelo contrário.

No presente estudo viu-se um índice geral de dentes cariados, perdidos ou obturados de 3,6 , e não houve uma correlação significativa $(p=0,3)$ entre o índice de CPO-d e Ceo-d e índice TF. O que corrobora outro estudo $^{13}$ realizado na África do Sul, no qual foram avaliadas 282 crianças com idades entre 10-15 anos que viveram desde o nascimento em três regiões onde a água de consumo era naturalmente fluoretada (LeeuGamka, 3.0; Kuboe 0.48; e Sanddrif 0.19 p.p.m. F).

Os dentes foram examinados e foi usado o índice CPO-d e o índice de Dean para avaliar fluorose dentária. A prevalência de fluorose (graus 2, 3, 4 e 5) foi de 42,5\% em Sanddrif, $50 \%$ em Kuboes e 95\% em LeeuGamka. Os graus de fluorose variaram significativamente entre os residentes na área de LeeuGamka $(3,0 \mathrm{ppm})$ e as outras duas regiões. A experiência de cárie na área de maior fluoretação na água de consumo foi significativamente maior do que nas outras duas localidades. Houve associação positiva entre as altas concentrações de flúor na água de consumo e a experiência de cárie, e não houve diferença entre índice CPO e fluorose nas áreas de baixa concentração de flúor ${ }^{13}$.

Em outra investigação ${ }^{15}$, avaliou-se 
1500 crianças selecionadas aleatoriamente em diferentes regiões com flúor in natura. Verificou-se uma prevalência de fluorose em $74,9 \%$ das crianças e $56,5 \%$ de cárie dentária. A prevalência de cárie e o CPO-d foram menores em regiões de baixa concentração de flúor. Houve uma prevalência moderada de fluorose e maior prevalência de cárie em áreas onde a concentração de flúor foi abaixo da concentração ideal ${ }^{14}$.

Já em outra pesquisa ${ }^{15}$, examinou-se a relação entre CPO-s, CFI (Índice de fluorose comunitária) e CPO-s individual, e índice de Dean. Em 1435 crianças de 12 anos de idade de 11 regiões diferentes de Porto Rico não houve diferença estatisticamente significativa entre o CPO e CFI por regiões. Observou-se que fluorose de moderada a severa esteve associada com altos índices de CPO, e que apesar de ser de vital importância controlar os índices de fluorose dentária, não há relação entre esse fato e a presença de cárie dentária ${ }^{15}$.

Alguns fatores podem ter prejudicado a análise de correlação entre experiência de cárie e graus de fluorose, como o pequeno número amostral. Como a população da área estudada é pequena, não houve a possibilidade de avaliar um número maior de indivíduos. Outro fator seria a falta de controle com pacientes residentes em áreas com baixa concentração de flúor na água de consumo. Associa-se a isto o uso do índice para analisar fluorose dentária. Pode-se usar o índice de Dean ou o índice TF, ficando difícil fazer uma comparação de resultados. Clinicamente, a fluorose pode se apresentar de diversas formas, dependendo das concentrações de fluoreto e do tempo de exposição. A desfiguração marrom-escurecida do esmalte com fluorose não é um resultado direto da amelogênese defeituosa, mas sim de corantes dos alimentos após a erupção dentária. Este é um dos pontos negativos para o uso do índice de Dean, que considera a presença destas manchas na avaliação da severidade. Por isso na presente pesquisa optou-se pelo índice TF. Portanto, o fato de cada pesquisa usar um índice diferente dificulta a comparação.

Para a situação periodontal do voluntário na pesquisa atual escolheu-se o PSR (Registro Periodontal Simplificado) por ser um sistema que permite o exame das estruturas periodontais de uma forma simples e rápida. Verificou-se que a maioria dos voluntários (69\%) apresentaram sangramento à sondagem. Ainda são poucos os estudos avaliando a relação entre doença periodontal em pacientes com fluorose dentária.

Porém, uma pesquisa ${ }^{16}$ avaliou 1029 voluntários com idades entre 15 e 74 anos e observou que gengivite e periodontite eram mais comuns entre mulheres $(65,9 \%$ e $32,8 \%$, respectivamente), e que, com o avançar da idade, a gengivite reduziu de $81 \%$ para $42,9 \%$. Já a periodontite, aumentou de $18 \%$ para $57,1 \%$. Os resultados sugerem que há uma forte relação entre a ocorrência de periodontite em regiões com altas concentrações de flúor ${ }^{16}$.

Com relação à percepção do paciente acerca da fluorose dentária, todos os pacientes pareciam estar cientes da presença das manchas, sentindo-se envergonhados com relação a seu sorriso. A percepção de saúde pode ser associada à qualidade de vida. Já se sabe que há diferenças culturais quanto à percepção individual do que seria esteticamente aceitável no sorriso. Ou seja, pessoas que vivem em áreas rurais teriam exigências estéticas diferentes daqueles que vivem em área urbana.

Pessoas que residem em áreas rurais se satisfazem mais facilmente a respeito de sua imagem própria do que aqueles que residem em regiões urbanas, uma vez que se considera que estes indivíduos sofrem mais pressão da mídia para que tenham um estereótipo perfeito.

Uma pesquisa ${ }^{17}$ mostrou que adolescentes que moram em áreas urbanas mostraram maior insatisfação com relação à aparência $(65,4 \%)$ do que aqueles residentes em áreas rurais $(52,6 \%)^{17}$. Contudo, outro estudo $^{18}$ não observou diferenças estatisticamente significativas entre percepção da imagem e localização geográfica.

Na presente pesquisa observou-se que as crianças mostram uma certa preocupação com relação à aparência de seus dentes. No 
entanto, poucos estudos realizados nesse tópico envolvem crianças ${ }^{18}$.

0 esmalte fluorótico não é mais resistente ao desenvolvimento da lesão cariosa; pelo contrário, é um esmalte mais quebradiço, o que faz com que o indivíduo portador de fluorose dentária tenha chance maior de apresentar lesões cariosas. Esse fato corrobora os resultados da presente pesquisa, uma vez que se viu que a maioria das crianças avaliadas apresentaram lesões de cárie ativa. Sendo necessário assim desenvolver projetos de prevenção de cárie dentária na comunidade da Vila do Brejo.

\section{CONCLUSÃO}

Conclui-se que as crianças residentes na região da Vila do Brejo apresentam cárie ativa, fluorose dentária em graus acima de 2 (índice $\mathrm{TF})$, e presença de sangramento gengival.

0 índice CPOD, no entanto, não revelou relação significativa com a fluorose dentária. Com relação à percepção viu-se que todos os voluntários estavam cientes da presença de manchas em seus dentes.

\section{REFERÊNCIAS}

1. Leal SD, Carvalho SF, Carvalho CAP. Conhecimento de alunos do Curso de Odontologia sobre o uso racional do flúor. Rev Odontol UNESP. 2015; 44(1):51-58.

2. Frazão P, Peres MA, Cury JA. Qualidade da água para consumo humano e concentração de fluoreto. Rev Saúde Pública. 2011; 45(5):964-73.

3. Sampaio FC, Ramm Von Der Fehr F, Arneberg P, Petrucci Gigante D, Hatloy A. Dental fluorosis and nutritional status of 6- to 11-year-old children living in rural areas of Paraíba, Brazil. Caries Res. 1999; 33(1):66-73. 4. Forte FDS, Freitas CHSM, Sampaio FC, Jardim MCAM. Fluorose dentária em crianças de Princesa Isabel, Paraíba. Pesqui Odontol Bras. 2001; 15(2):87-90.

5. Sampaio FC, Silva FDSCM, Silva ACB, Azevedo ATBM, Araújo DAM, Sousa EM. Natural fluoride levels in the drinking water, water fluoridation and estimated risk of dental fluorosis in a Tropical Region of Brazil. Oral Health Prev Dent. 2010; 8(1):71-5.

6. Souza CFM, Lima JFJ, Adriano MS, Carvalho
FG, Forte FD, Farias Oliveira $\mathrm{R}$, et al. Assessment of groundwater quality in a region of endemic fluorosis in the northeast of Brazil. Environ Monit Assess. 2013; 185(6):4735-43.

7. Narvai PC. Cárie dentária e flúor: uma relação do século XX. Ciênc Saúde Coletiva. 2000; 5(2):381-92.

8. Lima YBO, Cury JA. Ingestão de flúor por crianças pela água e dentifrício. Rev Saúde Pública. 2001; 35(6):576-81.

9. Moura MS, Barbosa PRR, Santos DLN, Dantas-Neta NB, Moura LFAD, Lima MDM. Vigilância epidemiológica da fluorose dentária em município de clima tropical com água de abastecimento público fluoretada. Ciênc Saúde Coletiva. 2016; 21(4):1247-54.

10. Fujibayashi SY, Archetti FB, Pizzatto S, Losso ES, Pizzatto E. Severidade de fluorose dental em um grupo de escolares. RSBO. 2011; 8(2):168-73.

11. Baskaradoss JK, Clement RB, Narayanan A. Prevalence of dental fluorosis and associated risk factors in 11-15 year old school children of Kanyakumari District, Tamilnadu, India: a cross sectional survey. Indian J Dent Res. 2008; 19(4):297-303.

12. Thylstrup A, Fejerskov 0 . Clinical appearance of dental fluorosis in permanent teeth in relation to histologic changes. Community Dent Oral Epidemiol. 1978; 6(6):315-28.

13. Grobleri SR, Louw AJ, Van Kotze TJ. Dental fluorosis and caries experience in relation to three different drinking water fluoride levels in South Africa. Int J Paediatr Dent. 2001; 11(5):372-9.

14. Shanthi M, Reddy BV, Venkataramana V, Gowrisankar S, Reddy BV, Chennupati S. Relationship between drinking water fluoride levels, dental fluorosis, dental caries and associated risk factors in 9-12 years old school children of Nelakondapally Mandal of Khammam District, Andhra Pradesh, India: a cross-sectional survey. J Int Oral Health. 2014; 6(3):106-10.

15. Elias-Boneta AR, Toro MJ, RivasTumanyan S, Murillo M, Orraca L, Encarnacion A, et al. Persistent oral health disparity in 12year-old hispanics: a cross-sectional study. BMC Oral Health. 2016; 16:10. 
16. Vandana KL, Reddy MS. Assessment of periodontal status in dental fluorosis subjects using community periodontal index of treatment needs. Indian J Dent Res. 2007; 18(2):67-71.

17. Triches R, Giugliani E. Insatisfação corporal em escolares de dois municípios da região Sul do Brasil. Rev Nutr. 2007; 20(2):119-28.

18. Welch C, Gross S, Bronner Y, DewberryMoore N, Paige D. Discrepancies in body image perception among fourth-grade public school children from urban, suburban, and rural Maryland. J Am Diet Assoc. 2004; 104(7):1080-5.

\section{CONTRIBUIÇÕES}

Consuelo Fernanda Macedo de Souza atuou na concepção, delineamento, análise e interpretação dos dados, escrita e revisão crítica do texto. Teodomiro Dutra de Abreu e Gabrielle Stella Dantas Barbosa participaram no delineamento, coleta de dados. Cynára Liane Jales Ataíde de Melo contribuiu na redação, análise e interpretação dos resultados. Maria Soraya Pereira Franco Adriano atuou na concepção, delineamento, análise e interpretação dos dados, redação e revisão crítica do texto.

\section{Como citar este artigo (Vancouver)}

Souza CMF, Abreu TD, Barbosa GSD, Melo CLJA, Adriano MSPF. Perfil de saúde bucal em escolares residentes em uma região endêmica de fluorose dental. REFACS [Internet]. 2018 [citado em inserir dia, mês e ano de acesso];6(2):181-188. Disponível em: inserir link de acesso. DOI: inserir link do DOI.

\section{Como citar este artigo (ABNT)}

SOUZA, C. M. F. et al. Perfil de saúde bucal em escolares residentes em uma região endêmica de fluorose dental. REFACS, Uberaba, v. 6, n. 2, p. 181-188, 2018. Disponível em: <inserir link de acesso>. Acesso em: inserir dia, mês e ano de acesso. DOI: inserir link do DOI.

\section{Como citar este artigo (APA)}

Souza, C. M. F.; Abreu, T. D.; Barbosa, G. S. D.; Melo, C. L. J. A. \& Adriano, M. S. P. F. (2018). Perfil de saúde bucal em escolares residentes em uma região endêmica de fluorose dental. REFACS, 6(2), 181-188. Recuperado em: inserir dia, mês e ano de acesso de inserir link de acesso. DOI: inserir link do DOI. 